\title{
N-POINT MEASURES OF POLYCRYSTALLINE MICROSTRUCTURE: MEASUREMENT, REPRESENTATION AND APPLICATIONS
}

\author{
BRENT L. ADAMS AND E. TURAN ONAT \\ Department of Mechanical Engineering, Yale University, New Haven, CT, USA
}

\section{Abstract}

Advanced models for engineering properties of polycrystalline materials require description of the spatial distribution of lattice phase and orientation. For this purpose the n-point statistical measures present a natural extension of the orientation distribution function, which is equivalent to the one-point measure of lattice orientation in single-phase microstructures. This paper describes the origin of the n-point measures in the context of statistical theory, and some aspects of their experimental determination. Fourier representation of the n-point measures in terms of tensorial basis functions is described. It is proposed that tensorial representations have some natural advantages over the ordinary representation using generalized spherical functions. An example of the application of occurrence of the n-point statistics of lattice orientation in a theory of creep in polycrystals is presented, and some limited comparisons with the uniform strain-rate and self-consistent theories are described.

\section{Origin of the N-Point Microstructural Measures}

Consider a comprehensive description of the microstructure of single-phase polycrystals, and its relation to the n-point statistical measures. The formalism extends to polyphase polycrystals, but only the single-phase condition is presently considered. The focus is upon the microstructure of a statistical ensemble of polycrystals, each element of which has identical external shape and processing history, but whose internal details exhibit an expected stochastic character.

Let $\mathbf{a}_{i}(i=1,2,3)$ denote basis vectors of the Bravais lattice in a perfect, stress-free reference crystal fixed in its orientation relative to an orthonormal laboratory frame defined by unit vectors $\mathbf{i}_{\mathbf{i}}$. Now assume that three independent basis vectors $\mathbf{e}_{\mathbf{i}}$ can be specified locally at each position in the polycrystal, corresponding to the same crystallographic vectors as $\mathbf{a}_{\mathbf{i}}$ in the reference crystal ${ }^{1}$. Thus $\mathbf{e}_{\mathbf{i}}(\mathbf{x})$ represents the local crystal lattice, which is generally rotated and distorted relative to the reference basis vectors. This choice must be made continuously throughout the polycrystal so that a oneto-one correspondence exists between $\mathbf{e}_{\mathrm{i}}(\mathbf{x})$ and $\mathbf{a}_{\mathrm{i}}$ according to the equation

$$
\mathbf{e}_{\mathrm{i}}(\mathbf{x})=\mathrm{D}_{\mathrm{ji}}(\mathbf{x}) \mathbf{a}_{\mathrm{j}} \text {. }
$$

This correspondence is not defined for points lying at the grain-boundary interface, and its specification in dislocated crystals requires passage to the continuum description of dislocations in which the number of dislocation lines goes to infinity as the Burger's vector goes to zero such that the product remains finite. ${ }^{2,3}$ The components $D_{\mathrm{ji}}(\mathbf{x})$ in 
expression (2.1) define a second-rank tensor field $\mathbf{D}(\mathbf{x})=\mathrm{D}_{\mathrm{ij}}(\mathbf{x}) \mathbf{i}_{\mathrm{i}} \otimes \mathbf{i}_{\mathrm{j}}$ which prescribes the affine transformation of an arbitrary lattice vector of the perfect, stressfree reference crystal, $\mathbf{u}$, to its corresponding local lattice vector, $\mathbf{v}(\mathbf{x})$, according to the relation

$$
\mathbf{v}(\mathbf{x})=\mathbf{D}(\mathbf{x}) . \mathbf{u} \text {. }
$$

This tensor field will be different for each element of the ensemble of polycrystals.

For the ensemble of polycrystals the probability density functional $P[D(x)]$ gives a complete description of the microstructure. $\mathrm{P}[\mathrm{D}(\mathbf{x})]$ is interpreted to be the probability that the tensor field lies between $D(x)$ and $D(x)+\delta D(x)$ in the ensemble. This functional is understood to represent the infinite limit of the n-point probability density functions,

$$
P[D(x)]=\lim _{n \rightarrow \infty} P_{n}\left(\mathbf{D}\left(x_{1}\right), \ldots, D\left(x_{n}\right)\right),
$$

which define the occurrence of $\mathbf{D}$ at $\mathbf{n}$ specified points in the ensemble of polycrystals. (For a comprehensive treatment of the statistics of continuous media, the monograph of Beran $^{4}$ is recommended.)

From an experimental viewpoint it is not possible to measure the entire tensor $\mathbf{D}$, even at a single location, except in fully annealed polycrystals. The problem stems from the fact that $\mathbf{D}$ reflects not only the local lattice orientation, but also the local elastic distortion of the Bravais lattice. Any experimental technique which uses sectioning to expose local positions in the polycrystal introduces new free surface, which will further distort the local lattice. For this reason we have generally been content to examine the pure rotation component of $\mathbf{D}$. These components are related through the well-known polar decomposition theorem; for example, there exists a unique decomposition of the form $\mathbf{D}=\mathbf{R} . \mathbf{A}$, where $\mathbf{A}$ is a symmetric tensor of second rank which carries the distortion of the lattice and $\mathbf{R}$ is a pure rotation. Hereafter, the functional of interest is $P[R(x)]$, and the $n$-point probability density functions of concern are $P_{n}\left(\mathbf{R}\left(x_{1}\right), \ldots, \mathbf{R}\left(x_{n}\right)\right)$.

It is useful to consider a related functional, $\mathrm{M}[\mathbf{Q}(\mathbf{x})]$, called the correspondence functional, which is defined by the Fourier transform of $P[R(x)]$ according to

$$
\mathrm{M}[\mathbf{Q}(\mathbf{x})]=\int_{R} \exp \left\{\mathrm{i} \int \mathbf{Q}(\mathbf{x}) \cdot \mathbf{R}(\mathbf{x}) \mathrm{dx}\right\} \mathrm{P}[\mathbf{R}(\mathbf{x})] \mathrm{dR}(\mathbf{x}) .
$$

The internal integration is over the volume occupied by the polycrystal, and $R$ denotes the infinite-dimensional function space defining all possible fields $\mathbf{R}(\mathbf{x})$ in the ensemble of polycrystals. In finite form this expression defines the $n$-point characteristic functions, $M_{n}\left(Q_{1}, \ldots, Q_{n}\right)$, according to 


$$
\mathbf{M}_{\mathbf{n}}\left(\mathbf{Q}_{1}, \ldots, \mathbf{Q}_{\mathbf{n}}\right)=\int_{\mathrm{SO}(3)} . \int_{\mathrm{SO}(3)} \exp \left\{\mathrm{i} \sum_{\mathrm{k}=1}^{\mathrm{n}} \mathbf{Q}_{\mathrm{k}} . \mathbf{R}_{\mathbf{k}}\right\} \mathbf{P}_{\mathbf{n}}\left(\mathbf{R}_{1}, . ., \mathbf{R}_{\mathbf{n}}\right) \mathrm{d} \mathbf{R}_{1} \ldots \mathrm{dR}_{\mathbf{n}} \text {. }
$$

The tensors $\mathbf{Q}_{\mathbf{k}}\left(=\mathbf{Q}\left(\mathbf{x}_{\mathrm{k}}\right)\right)$ and $\mathbf{R}_{\mathbf{k}}\left(=\mathbf{R}\left(\mathbf{x}_{\mathrm{k}}\right)\right)$ both belong to the special orthogonal group of rotations, SO(3). In the limit as $n$ approaches infinity equations (2.4) and (2.5) are equivalent. Expansion of the exponential function appearing in the integrand of (2.5) leads to the following expression:

$$
\begin{aligned}
& \mathbf{M}_{\mathrm{n}}\left(\mathbf{Q}_{1}, \ldots, \mathbf{Q}_{\mathrm{n}}\right)=1+\mathrm{i} \sum_{\mathrm{k}=1}^{\mathrm{n}} \int_{\mathrm{SO}(3)}\left(\mathbf{Q}_{\mathrm{k}} \cdot \mathbf{R}_{\mathrm{k}}\right) \mathrm{P}_{1}\left(\mathbf{R}_{\mathrm{k}}\right) \mathrm{d} \mathbf{R}_{\mathrm{k}} \\
& -\frac{1}{2} \sum_{j=1}^{n} \sum_{k=1}^{n} \int_{S O(3)} \int_{S O(3)}\left(\mathbf{Q}_{j} \cdot \mathbf{R}_{j}\right) \cdot\left(\mathbf{Q}_{k} \cdot \mathbf{R}_{k}\right) P_{2}\left(\mathbf{R}_{j}, \mathbf{R}_{k}\right) d \mathbf{R}_{j} d \mathbf{R}_{k} \\
& + \text { higher-order terms }
\end{aligned}
$$

We emphasize the hierarchy of probability density functions required to evaluate successive terms in the series expansion. After the constant term on the right hand side of (2.6) appears a set of $n$ terms containing mean values of the rotation tensor, evaluated at $\mathbf{n}$ distinct points in the ensemble of polycrystals. For statistically homogeneous polycrystals each of the functions necessary to evaluate this second term coincides with the well-known orientation distribution function.. Next in equation (2.6) occurs $\mathrm{n}^{2}$ terms containing two-point moments of lattice orientation. In the limit that $\mathbf{n}$ becomes very large (i.e., as the n-point characteristic function becomes the characteristic functional) it is clear that the two-point coherence function of lattice orientation is required. The two-point coherence function is the fourth-rank tensor

$$
\left\langle\mathbf{R}_{1} \mathbf{R}_{2}\right\rangle=\int_{\mathrm{SO}(3)} \int_{\mathrm{SO}(3)} \mathbf{R}_{1} \mathbf{R}_{2} \mathrm{P}_{2}\left(\mathbf{R}_{1}, \mathbf{R}_{2}\right) \mathrm{d} \mathbf{R}_{1} \mathbf{d} \mathbf{R}_{2},
$$

where we think of $\mathbf{R}_{1}$ and $\mathbf{R}_{2}$ as continuous functions of $\mathbf{x}_{1}$ and $\mathbf{x}_{2}$. For a statistically homogeneous ensemble of polycrystals it is clear that the two-point coherence function, as well as the two-point probability density function $\mathrm{P}_{2}$, are functions of the difference $\mathbf{x}_{2}-\mathbf{x}_{1}$ only. Adams, et. al. ${ }^{5}$ and Adams, Wang and Morris ${ }^{6}$ previously called the twopoint probability density function the two-point orientation coherence function.

As the higher-order moments are considered in relation (2.6) higher-order statistics of the microstructure are required. In the passage to the characteristic functional $\mathrm{M}[\mathrm{Q}(\mathrm{x})]$, the continuous n-point coherence functions, $\left\langle\mathbf{R}_{1} \ldots \mathbf{R}_{\mathrm{n}}\right\rangle$, occur in the series expansion, and computation of these requires the n-point probability density functions, $P_{n}\left(R_{1}, \ldots, R_{n}\right)$. This is the formal basis for the occurrence of $n$-point statistical functions in the description of microstructures. We emphasize that a description of the functional $P[R(x)]$, or equivalently the characteristic functional $M[Q(x)]$, requires the infinite-point statistics of lattice orientation for the ensemble of polycrystals. Current state-of-the-art experimental and computational capabilities will certainly limit the characterization of microstructures to the second- or third-order moments in the forseeable future. A complete experimental characterization of the microstructure is generally not practical. The familiar orientation distribution function fully defines the first moment of the complete functional, and the recently introduced two-point 
statistics fixes the second-order moments. One, two and three-point statistics of lattice orientation can be obtained from ordinary oblique sectioning methods of stereology when a suitable microdiffraction technique is available. Extensions to four, or higherpoints require serial sectioning.

\section{Tensorial Representation of the N-Point Measures}

In our previous work ${ }^{5-7}$ we extended the traditional Fourier representation of the orientation distribution function, based upon generalized spherical functions, to consider the two-point orientation coherence function. Here we propose a new Fourier representation with tensorial character. There is good reason to consider a departure from the traditional basis functions used in texture analysis. It is known from the mechanics of solids that parameters representing the internal state of a material must have tensorial character ${ }^{8}$. Consequently, when we desire to study relationships connecting mechanical properties to microstructure, it is most natural to use tensorial basis functions since the components and scales of microstructure can then be directly correlated with measured behavior. Tensorial representation of microstructure also lends itself to a very useful geometrical interpretation which substantially clarifies the role of the crystallographic symmetry group in forming basis functions.

In the presentation which follows the tensorial Fourier representation of the orientation distribution function is first described, with special emphasis on lattices exhibiting cubic symmetry. The extension to the n-point statistics of microstructure is then considered.

\subsection{Construction of the Irreducible Tensorial Representation}

Completely symmetric and traceless tensors of rank $\mathrm{r}$ are known to be irreducible (cf. Gel'fand, Minlos and Shapiro ${ }^{9}$ ). The components of such tensors have the following two properties:

$$
\mathrm{t}_{i_{1} i_{2} \ldots i_{\mathrm{r}}}=\mathrm{t}_{\mathrm{p}\left(i_{1} i_{2} \ldots i_{\mathrm{r}}\right)}, \mathrm{t}_{i} i i_{3} \ldots i_{\mathrm{r}}=0 \text {, }
$$

where $\mathrm{p}$ denotes permutation, and repeated occurrence of indices implies summation from 1 to 3 according to the Einstein convention.

It has been known since the times of Sylvester and Maxwell that such a tensor defines uniquely a bundle of r-lines (cf. Courant and Hilbert ${ }^{10}$ ). By assigning directions to a bundle of r-lines passing through a common origin, it is possible to associate the irreducible tensor of rank $r$ with $r$ vectors of equal length. For example, the components of a fourth-rank irreducible tensor can be expressed as

$$
\begin{aligned}
\mathrm{t}_{i j k l}= & \sum_{\mathrm{p}(\alpha \beta \gamma \delta)} \mathrm{v}_{i}^{\alpha} \mathrm{v}_{j}^{\beta} \mathrm{v}_{k}^{\gamma} \mathrm{v}_{l}^{\delta} \\
& -\frac{1}{7}\left(\mathbf{v}^{\alpha} \cdot \mathrm{v}^{\beta}\right)\left(\delta_{i j} \mathrm{v}_{k}^{\gamma} \mathrm{v}_{l}^{\delta}+\delta_{i k} \mathrm{v}_{j}^{\gamma} \mathrm{v}_{l}^{\delta}+\delta_{i l} \mathrm{v}_{j}^{\gamma} \mathrm{v}_{k}^{\delta}+\delta_{j k} \mathrm{v}_{i}^{\gamma} \mathrm{v}_{l}^{\delta}+\delta_{j l} \mathrm{v}_{i}^{\gamma} \mathrm{v}_{k}^{\delta}+\delta_{k l} \mathrm{v}_{i}^{\gamma} \mathrm{v}_{j}^{\delta}\right) \\
& +\frac{1}{7 \times 5}\left(\mathbf{v}^{\alpha} \cdot \mathrm{v}^{\beta}\right)\left(\mathbf{V}^{\gamma} \cdot \mathrm{v}^{\delta}\right)\left(\delta_{i j} \delta_{k l}+\delta_{i k} \delta_{j l}+\delta_{i l} \delta_{j k}\right)
\end{aligned}
$$

where any four vectors satisfying the condition $\left|\mathbf{V}^{\alpha}\right|=\left|\mathbf{V}^{\beta}\right|=\left|\mathbf{V}^{\gamma}\right|=\left|\mathbf{V}^{\delta}\right|$ may be used. ${ }^{11}$ Expressions of similar nature are easily constructed for traceless and symmetric tensors of any rank. Whenever a vector is exchanged with its opposite, the tensor itself changes sign as can be readily seen in expression (3.2).

The irreducible tensor to of rank $\mathrm{r}$ can be formally shown to generate, by tensor transformation, $(2 r+1)$ linearly independent functions defined on $\mathrm{SO}(3)$ : 


$$
\mathrm{t}_{j_{1} j_{2} \ldots j_{\mathbf{r}}}(\mathbf{R})=\mathbf{R}_{i_{1} j_{1}} \mathbf{R}_{i_{2} j_{2} \ldots} \ldots \mathbf{R}_{i_{\mathrm{r}} j_{\mathbf{r}}} \mathbf{t}_{i_{1} i_{2} \ldots i_{\mathbf{r}}}\left(\text { or } \mathbf{t}=\mathrm{P}_{\mathbf{R}} \mathbf{t}^{\mathrm{o}}\right) \text {. }
$$

Moreover, these functions can be expressed as linear combinations of the generalized spherical functions frequently used in texture analysis. It can also be shown that if to has the symmetry of the lattice, then the functions $\mathbf{t}$ will also carry the lattice symmetry. In other words, if $L \subset \mathrm{SO}(3)$ is the finite symmetry subgroup of the lattice of interest, then

$$
\mathbf{t}^{\mathbf{o}}=\mathrm{P}_{\mathbf{Q}} \mathbf{t}^{\mathbf{o}}, \quad \mathrm{Q} \in L .
$$

Furthermore, the functions $t$ have the property

$$
\mathrm{t}_{j_{1} j_{2} \ldots j_{\mathrm{r}}}(\mathbf{R} . \mathbf{Q})=\mathrm{t}_{j_{1} j_{2} \ldots j_{\mathbf{r}}}(\mathbf{R}) \text {. }
$$

\subsection{Irreducible Tensors with Cubic Symmetry}

There exists only three basic groups of vectors which can be combined to generate tensors exibiting the pure rotational symmetries of the cubic lattice: (a) three orthogonal vectors joining the centroid of a cube to the middle of the faces, (b) four vectors emanating from the centroid to the common corners of a face, and (c) six vectors emanating from the centroid to the midpoint of half of the edges of the cube. These are illustrated in following figure:

a

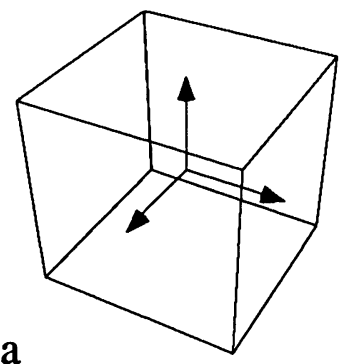

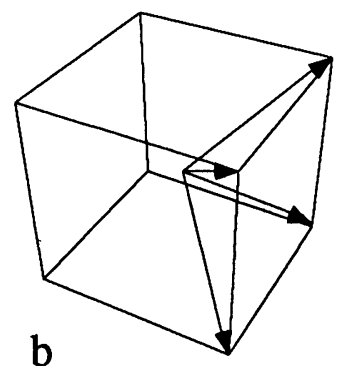

b

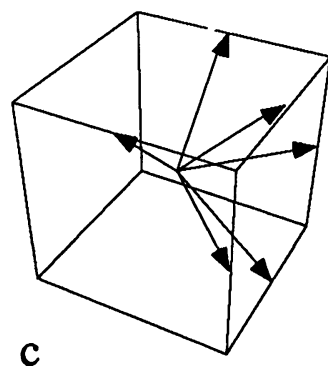

c

Tensors generated by odd multiples of bundles a and $\mathrm{c}$ do not exhibit cubic symmetry. All multiples of bundle $b$ exhibit cubic symmetry. Tensors of odd rank are generated by combinations having an odd number of bundles a and of $c$. The next figure shows the graphical representation of the first few tensors exhibiting cubic symmetry.
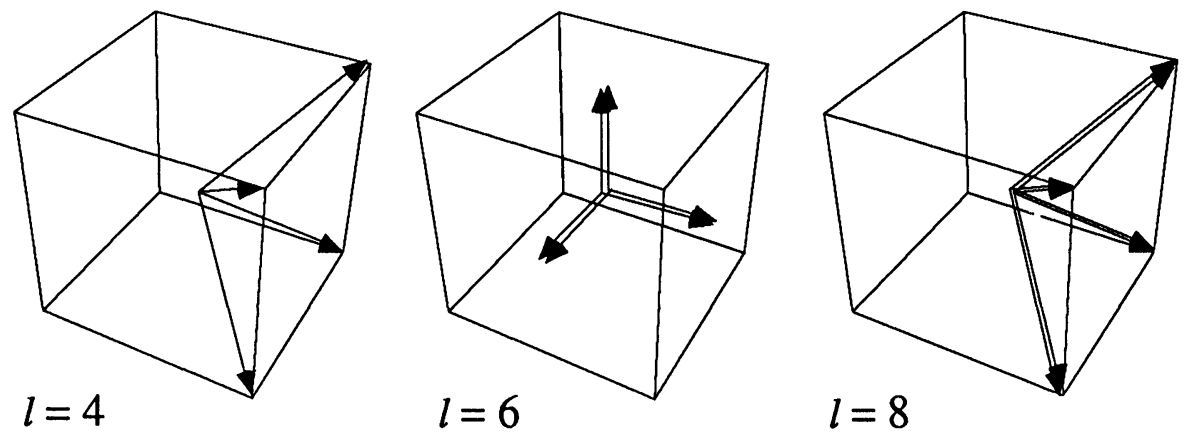

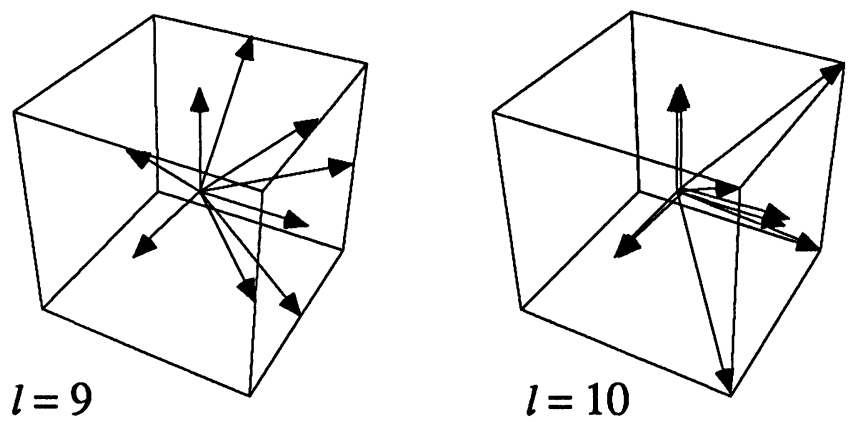

It is easily shown by the graphical construction that no tensors of rank $r=1,2,3,5,7,11$, ...etc. exist. When $r=12$ three distinct tensors are found by the graphical construction which have cubic symmetry. These are shown in the next figure.
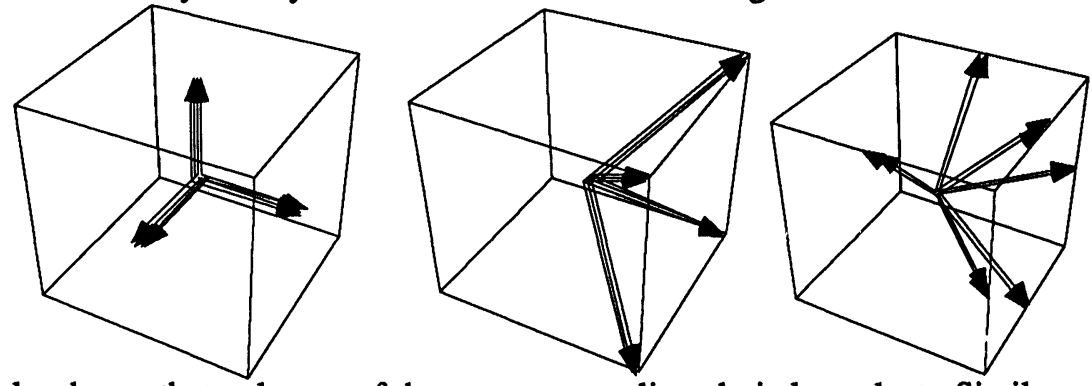

It can be shown that only two of these tensors are linearly independent. Similar analysis with with tensors of rank $r=12$ through 24 shows that the number of linearly independent bundles coincides with the number of independent generalized spherical functions in the traditional analysis (cf. Bunge ${ }^{12}$ ).

\subsection{Tensorial Representation of the Orientation Distribution Function}

The orientation distribution function (odf) is a function defined over the set $\mathbf{E}$ of physically distinctive orientations of the crystal lattice: odf $=f: E \rightarrow R$ where $R$ is the real line. $f(R) d R$ expresses the volume fraction of crystallites in the polycrystal who have orientations in the neighborhood $\mathrm{d} \mathbf{R}$ about $\mathbf{R}$ where $\mathbf{R} \in \mathbf{E}$ expresses the rotation of the reference lattice. We shall generally work with an auxiliary function $\hat{f}: S O(3) \rightarrow R$ which has the following property:

$$
\hat{\mathrm{f}}(\mathbf{R} . \mathbf{Q})=\hat{\mathrm{f}}(\mathbf{R}) \quad \text { for all } \mathbf{Q} \in L \text { and } \mathbf{R} \in \mathrm{SO}(3) \text {. }
$$

The tensorial Fourier representation of $\hat{f}(R)$ for cubic polycrystals is given by

$$
\hat{f}(\mathbf{R})=C_{o}+C_{i j k l} t_{i j k l}(\mathbf{R})+C_{i j k l m n} t_{i j k l m n}(\mathbf{R})+\ldots,
$$

where the series contains all non-zero tensors exhibiting cubic symmetry.

It is easily demonstrated that the coefficients of the series, $C_{i j k l . . .}$, can be taken to be completely symmetric and traceless in their indices in the same manner prescibed in (3.1). Furthermore, some reflection reveals that if the reference lattice is rotated by $\mathbf{S}$ the odf changes according to the prescription (cf. Onat and Leckie ${ }^{11}$ ) 


$$
\hat{\mathrm{f}}(\mathbf{R}) \rightarrow \mathrm{PS}_{\mathbf{S}} \hat{\mathrm{f}}(\mathbf{R})=\hat{\mathrm{f}}\left(\mathbf{S}^{-1} \cdot \mathbf{R}\right)=\hat{\mathrm{f}}\left(\mathbf{S}^{\mathrm{T}} \cdot \mathbf{R}\right) .
$$

This relation makes it clear that the series coefficients, $C_{i j k l . .}$, are tensorial in the sense that they transform according to

$$
\left(\mathrm{C}_{\mathrm{o}}, \mathrm{C}_{i j k l}, \ldots\right) \rightarrow\left(\mathrm{C}_{\mathrm{o}}, \mathrm{S}_{i m} \mathrm{~S}_{j n} \mathrm{~S}_{k o} \mathrm{~S}_{l p} \mathrm{C}_{m n o p}, \ldots\right) .
$$

Thus, the symmetry of the lattice is carried in the tensorial basis functions and the sample symmetry is carried in the series coefficients, which also transform like tensors.

\subsection{Tensorial Representation of the N-Point Microstructural Measures}

The n-point orientation coherence function, $\mathrm{P}_{\mathbf{n}}\left(\mathbf{R}_{1}, \ldots, \mathbf{R}_{\mathbf{n}}\right)$, expresses the probability density of occurrence of orientations at $n$-points in the ensemble. $P_{n}\left(R_{1}, \ldots, \mathbf{R}_{n}\right)$ $\mathrm{d} \mathbf{R}_{1} \ldots \mathrm{d} \mathbf{R}_{\mathrm{n}}$ gives the fraction of experimental trials, positioned at $x_{1}, \ldots, x_{n}$ in the ensemble, obtain lattice orientations in the neighborhoods $d \mathbf{R}_{1}, \ldots, d \mathbf{R}_{n}$ surrounding $\mathbf{R}_{1}$, $\ldots, \mathbf{R}_{\mathbf{n}}$. Clearly the $\mathrm{n}$-point orientation coherence function maps elements of an $n^{\text {th }}$ order

product space of $S O(3)$ to the real line: $P_{n}: S O(3)_{1} \times S O(3)_{2} \times \ldots \times S O(3)_{n} \rightarrow R$ Given than an orthonormal basis is known for $S O(3)$, the tensorial Fourier representation of $P_{n}$ is expressed in terms of $n^{\text {th }}$ order products of the irreducible tensors carrying the lattice symmetry (cf. Gel'fand, Minlos and Shapiro ${ }^{9}$ ). A few moments of reflection suggests a series of the form

$$
\begin{aligned}
\mathrm{P}_{\mathbf{n}}\left(\mathbf{R}_{1}, \ldots, \mathbf{R}_{\mathbf{n}}\right)= & \mathrm{D}_{\mathrm{o}}+\mathrm{D}_{i j k l} \widetilde{\mathrm{t}}_{i j k l}\left(\mathbf{R}_{1}, \ldots, \mathbf{R}_{\mathbf{n}}\right) \\
& +\mathrm{D}_{i j k l m n} \tilde{\mathrm{t}_{i j k l m n}}\left(\mathbf{R}_{1}, \ldots, \mathbf{R}_{\mathbf{n}}\right) \\
& +\mathrm{D}_{i j k l m n o p} \tilde{\mathrm{t}}_{i j k l m n o p}\left(\mathbf{R}_{1}, \ldots, \mathbf{R}_{\mathbf{n}}\right)+\ldots,
\end{aligned}
$$

where the tensors, $\tilde{t}$, are linear combinations of the lattice symmetric $\dot{t}$ functions; i.e.,

$$
\begin{gathered}
\tilde{\mathrm{t}}_{i j k l}\left(\mathbf{R}_{1}, \ldots, \mathbf{R}_{\mathrm{n}}\right)=\sum_{q=1}^{\mathrm{n}} \mathrm{a}_{q} \mathrm{t}_{i j k l}\left(\mathbf{R}_{q}\right), \\
\tilde{\mathrm{t}}_{i j k l m n}\left(\mathbf{R}_{1}, \ldots, \mathbf{R}_{\mathrm{n}}\right)=\sum_{q=1}^{\mathrm{n}} \mathrm{b}_{q} \mathrm{t}_{i j k l m n}\left(\mathbf{R}_{q}\right), \\
\widetilde{\mathrm{t}}_{i j k l m n o p}\left(\mathbf{R}_{1}, \ldots, \mathbf{R}_{\mathrm{n}}\right)=\sum_{q=1}^{\mathrm{n}} \mathrm{c}_{q} \mathrm{t}_{i j k l m n o p}\left(\mathbf{R}_{q}\right)+\sum_{q=1}^{\mathrm{n}} \sum_{r=1}^{\mathrm{n}} \mathrm{d}_{q r} \mathrm{t}_{i j k l}\left(\mathbf{R}_{q}\right) \mathrm{t}_{m n o p}\left(\mathbf{R}_{r}\right) .
\end{gathered}
$$

The coefficients $D_{\mathrm{ijkl} . . .}$ in (3.10) are considered to be continuous functions of $\mathbf{x}_{1}, \ldots, \mathbf{x}_{\mathbf{n}}$.

\section{Statistical Theory for Creep Behavior of Polycrystals}

Consider the occurrence of the n-point orientation coherence functions in creep constitutive modeling of polycrystals. Of interest is a class of single crystal creep behavior where the Eulerian strain-rate tensor, $\varepsilon$, is related to the deviatoric Cauchy stress, $\mathbf{S}$, through a nonlinear constitutive law which may be expressed as $\varepsilon=\mathbf{M}(\mathbf{R}, \mathbf{s})$.s. $M$ denotes the fourth-rank tensor of creep compliances which is a function of crystal orientation and stress. The power-law slip formulation of Hutchinson ${ }^{13}$ for creeping polycrystals is thought of here since it derives from a convex stress potential which 
assures that the inverse equation, $s=\mathbf{N}(R, \varepsilon) \cdot \varepsilon$, exists $\left(\mathbf{N}=\mathbf{M}^{-1}\right) . \mathbf{N}$ is called the tensor of creep moduli.

Consider the polycrystal to be an extensive, space-filling aggregate of crystallites which are perfectly bonded at their interfaces. We assume the outer boundary of the polycrystal extends to infinity whereupon a uniform velocity gradient is imposed. The local velocity gradient at every interior point is allowed to deviate from the uniform boundary value; it is assumed, however, that the local tensor does not deviate 'too far' from the uniform value. This assumption is valid for most single-phase polycrystals prior to the onset of tertiary creep. Essentially, we shall be concerned with relaxation of the uniform strain-rate condition imposed in Taylor-like models. Elastic contributions to the local strain-rate are neglected. The microstructure considered is that of the ensemble of polycrystals discussed in section 2. The n-point orientation coherence functions are assumed to exhibit spatial homogeneity. Interchanging volume averages with ensemble averages, according to the ergodic hypothesis, is assumed to be valid. The ensemble is also assumed to exhibit a finite coherence length such that decoupling of the n-point statistics occurs when points are separated by distances exceeding the coherence length. Further details of the theory are given elsewhere. ${ }^{14}$

Fields of stress and strain-rate are required to satisfy, respectively, the stress equilibrium and strain-rate compatibility conditions. Application of the two-Green's function method of Molinari et.al. ${ }^{15}$ to the stress equilibrium equations results in localization relations of the form

$$
\varepsilon=\bar{\varepsilon}+\Gamma^{*}[\delta \mathbf{N} . \varepsilon-\langle\delta \mathbf{N} . \varepsilon\rangle], \omega=\bar{\omega}+\Lambda^{*}[\delta \mathbf{N} . \varepsilon-\langle\delta \mathbf{N} . \varepsilon\rangle],
$$

where $\Gamma^{*}$ and $\Lambda^{*}$ denote linear operators expressing convolution integration of symmetric and antisymmetric second-derivatives of Green's functions over the volume of the polycrystal. $\delta \mathbf{N}=\mathbf{N}-\mathbf{N o}^{\circ}$ is the difference between the local creep modulus and a constant reference modulus, $\mathrm{N}^{\circ}$, which is conveniently defined to be the volume (ensemble) averaged value of $\mathbf{N}$ evaluated at the uniform strain-rate $\bar{\varepsilon}$ The operators $\Gamma^{*}$ and $\Lambda^{*}$ depend only upon the constant reference modulus tensor and the boundary conditions of the problem. What is important about relations (4.1) is that the local strain-rate or spin tensors deviate from the uniform values at the boundary by a convolution of the local values of these same tensors over the volume of the polycrystal. The highly implicit character of these relations is noted.

The theory next makes use of the assumption that the local velocity gradients do not vary too far from the uniform boundary value; this permits the local creep modulus to be expressed in a Taylor-series expanded about the uniform value of strain-rate. Introducing this expansion into (4.1), and iterating to remove the implicit character of the integral convolution gives rise to an asymptotic form of the localization relations.

What is of interest in the statistical theory is not localization relations for explicit microstructures, but relations pertinent to the ensemble. In particular, we give relations for partial ensemble averages of the local strain-rate and spin, given that condition $\phi$ occurs in the neighborhood of the point of interest. In its simplest form condition $\phi$ would specify lattice orientation $\mathbf{R}$ in the local particle. More complex conditions would specify additional microstructural details in the neighborhood of the local particle (e.g., lattice orientations in the neighboring grains). The partial ensemble average of () is denoted as $\langle() \mid \phi\rangle$. The interpretation is that we take averages over a partial ensemble satisfying condition $\phi$. The expressions for $\langle\varepsilon \mid \phi\rangle$ and $\langle\omega \mid \phi\rangle$ are 


$$
\begin{aligned}
& \langle\varepsilon \mid \phi\rangle=\bar{\varepsilon}+\left\{\Gamma^{*}[\overline{\delta \mathbf{N}}|\phi\rangle-\langle\overline{\delta N}\rangle]+\Gamma^{*}\left[\overline{\delta \mathbf{N}} \cdot \Gamma^{*} \overline{\delta \mathbf{N}}|\phi\rangle-\langle\overline{\delta N} \cdot \Gamma * \overline{\delta N}\rangle\right.\right. \\
& \left.+\left\langle\overline{\delta \mathbf{N}^{\prime}} \cdot \bar{\varepsilon} \cdot \Gamma^{*} \overline{\delta \mathbf{N}} \mid \phi\right\rangle-\left\langle\overline{\delta \mathbf{N}^{\prime}} \cdot \bar{\varepsilon} \cdot \Gamma^{*} \overline{\delta \mathbf{N}}\right\rangle+\langle\overline{\delta N}\rangle \cdot \Gamma * \sqrt{\delta \mathbf{N}}\right\rangle
\end{aligned}
$$

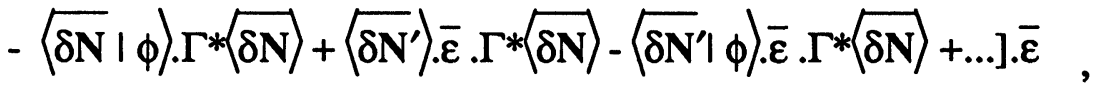

$$
\begin{aligned}
& \langle\omega \mid \phi\rangle=\bar{\omega}+\{\Lambda *[\overline{\delta \mathbf{N}}|\phi\rangle-\overline{\delta \mathbf{N}}\rangle]+\Lambda *\left[\sqrt{\delta \mathbf{N}} \cdot \Lambda^{*} \overline{\delta \mathbf{N}}|\phi\rangle-\left\langle\overline{\delta \mathbf{N}} \cdot \Lambda^{*} \overline{\delta \mathbf{N}}\right\rangle\right. \\
& +\left\langle\overline{\delta \mathbf{N}^{\prime}} \cdot \bar{\varepsilon} \cdot \Lambda^{*} \overline{\delta \mathbf{N}} \mid \phi\right\rangle-\left\langle\overline{\delta \mathbf{N}^{\prime}} \cdot \bar{\varepsilon} \cdot \Lambda^{*} \overline{\delta \mathrm{N}}\right\rangle+\langle\overline{\delta \mathrm{N}}\rangle \cdot \Lambda *\langle\overline{\delta \mathrm{N}}\rangle
\end{aligned}
$$

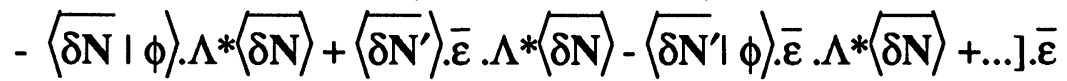

where $\overline{\delta N}$ denotes the polarized creep modulus evaluated at $\bar{\varepsilon}$ and $\overline{\delta N^{\prime}}$ denotes the first derivative of the polarized modulus, with respect to components of the strain-rate tensor, evaluated at the uniform condition.

When the n-point orientation coherence functions are known elements of the series in (4.2) and (4.3) can be calculated. For example

$$
\langle\overline{\delta \mathbf{N} \mid} \mathbf{R}\rangle_{i j k l}=\int_{\mathrm{SO}(3)} \overline{\delta \mathbf{N}}_{i j k l}\left(\mathbf{R}_{1}\right) \mathrm{P}_{2}\left(\mathbf{R}_{1} \mid \mathbf{R}\right) \mathrm{d} \mathbf{R}_{1}
$$

and

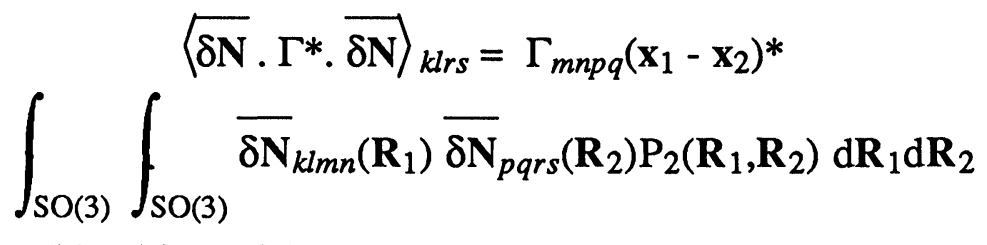

The conditional form of the two-point orientation coherence function, appearing in (4.5) is related to the two-point function by $\mathrm{P}_{2}\left(\mathbf{R}_{1}, \mathbf{R}\right)=\mathrm{P}_{2}\left(\mathbf{R}_{1} \mid \mathbf{R}\right) \mathrm{P}_{1}(\mathbf{R})$

When only the first-order statistics are carried in the statistical localization relations, (4.2) and (4.3) reduce to the well-known Taylor condition; i.e., $\langle\varepsilon \mid \phi\rangle=\bar{\varepsilon}$ and $\langle\omega \mid \phi\rangle=\bar{\omega}$. If decoupling can be assumed to occur after two-point statistics are considered (i.e., if three- and higher-point statistics can be adequately expressed in terms of the two-point orientation coherence function) then the uniform strain-rate assumption is replaced with a first-order correction for local heterogeneity of the strainrate and spin fields.

The theory is now applied to face-centered-cubic polycrystals exhibiting random lattice orientation, no spatial coherence between near-neighboring crystallites, and an isotropic and uniform coherence $d_{c}$. Recent experimental studies of Wang, Morris and Adams ${ }^{16}$ have demonstrated that such microstructures exist in grain-refined cast alloy 1100 aluminum. We shall consider only first-order corrections to the uniform strainrate theory; thus, only two-point statisitics of lattice orientation are carried in the theory. The statistical form of the localization equations reduces to 


$$
\begin{aligned}
& \langle\varepsilon \mid \mathbf{R}\rangle=\bar{\varepsilon}+\left\{\Gamma^{*}[\langle\overline{\delta \mathbf{N}} \mid \mathbf{R}\rangle-\langle\overline{\delta N}\rangle] \cdot \bar{\varepsilon},\right. \\
& \langle\omega \mid \mathbf{R}\rangle=\bar{\omega}+\left\{\Lambda^{*}[\langle\overline{\delta \mathbf{N}} \mathbf{R}\rangle-\langle\overline{\delta \mathbf{N}}\rangle] \cdot \bar{\varepsilon}\right.
\end{aligned}
$$

where the condition $\phi$ has been taken to be the occurence of lattice orientation $\mathbf{R}$ in the local crystallite. For the microstructure of interest the two-point statistics have the form

$$
\mathrm{P}_{2}\left(\mathbf{R}^{\prime} \mid \mathbf{R}\right)=\mathrm{h}(\mathrm{r}) \delta\left(\mathbf{R}^{\prime}-\mathbf{R}\right)+(1-\mathrm{h}(\mathrm{r})) \mathrm{P}_{1}\left(\mathbf{R}^{\prime}\right)
$$

where $r=\left|\mathbf{x}^{\prime}-\mathbf{x}\right|, \delta\left(\mathbf{R}^{\prime}-\mathbf{R}\right)$ is the Dirac function centered at $\mathbf{R}$, and $h(r)$ is a monotonically decreasing function defined over the interval $\left[0, d_{c}\right]$ which has a value of 1 when $r=0$ and a value of 0 when $r=d_{c}$. ( $\left(d_{c}\right.$ is the coherence distance in the material.) For this material the homogeneous reference medium is isotropic and hence the reference creep modulus and the associated Green's function convolutions are also isotropic.

Application of relations (4.6) and (4.7) to obtain a relationship for the tensile constitutive behavior of isotropic polycrystals gives an expression of the form

$$
\bar{\varepsilon}=\dot{\gamma}_{0}\left(\bar{S} / \bar{S}_{0}\right)^{n}
$$

where $\dot{\gamma}_{0}$ is the reference slip-rate in the crystallites (when a slip system sees a resolved shear stress of $\tau_{0}$ a slip rate of $\dot{\gamma}_{0}$ is observed), $\mathrm{n}$ is the inverse strain-rate sensitivity parameter, and $\bar{S}_{\mathrm{o}}$ is the tensile reference stress which completely specifies the behavior of the polycrystal. The following figure illustrates the results of the present theory compared with the uniform strain-rate upper-bound and Hutchinson's self-consistent estimate. 13

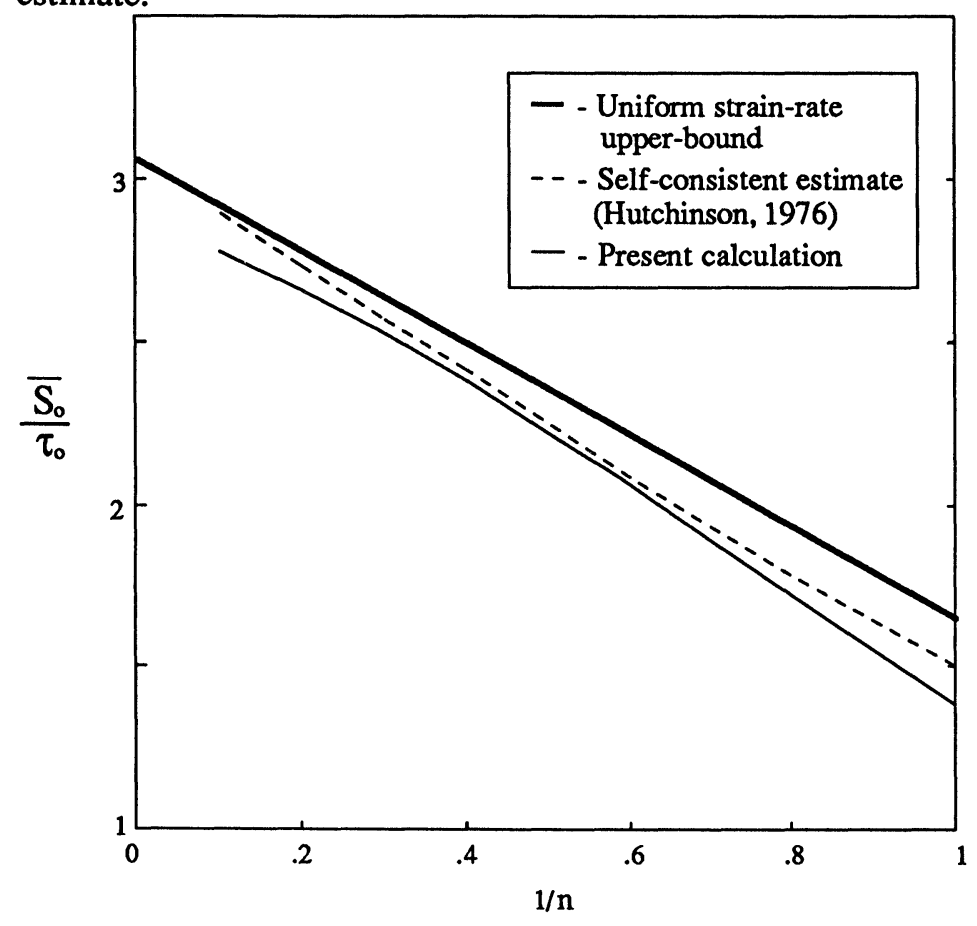


The softer response of the tensile reference stress, particularly in the rate-independent limit $(1 / n=0)$, is of considerable interest. Estimates of the initial flow field for the uniform strain-rate is shown in the next figure.

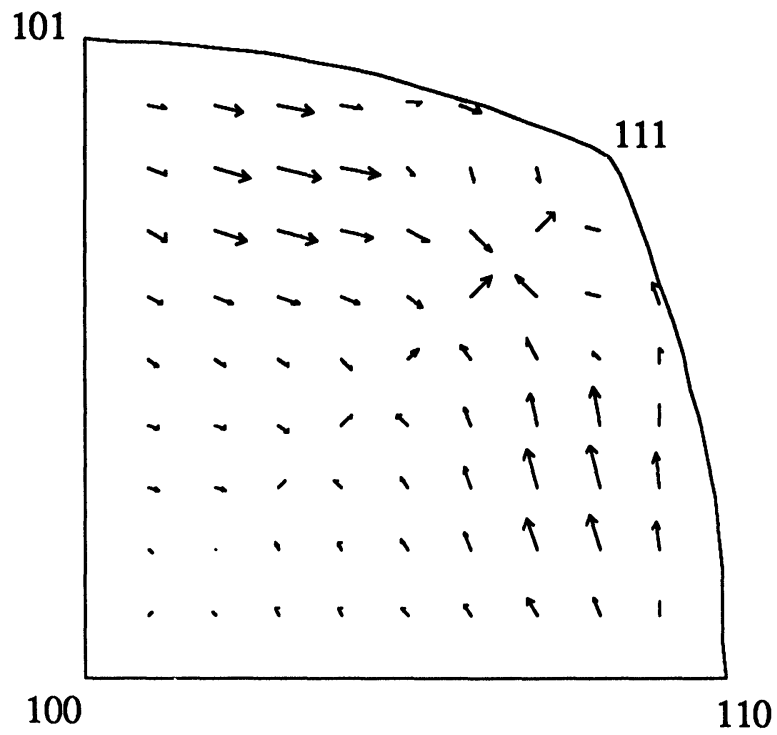

Estimates for the present heterogeneous field theory are shown next .

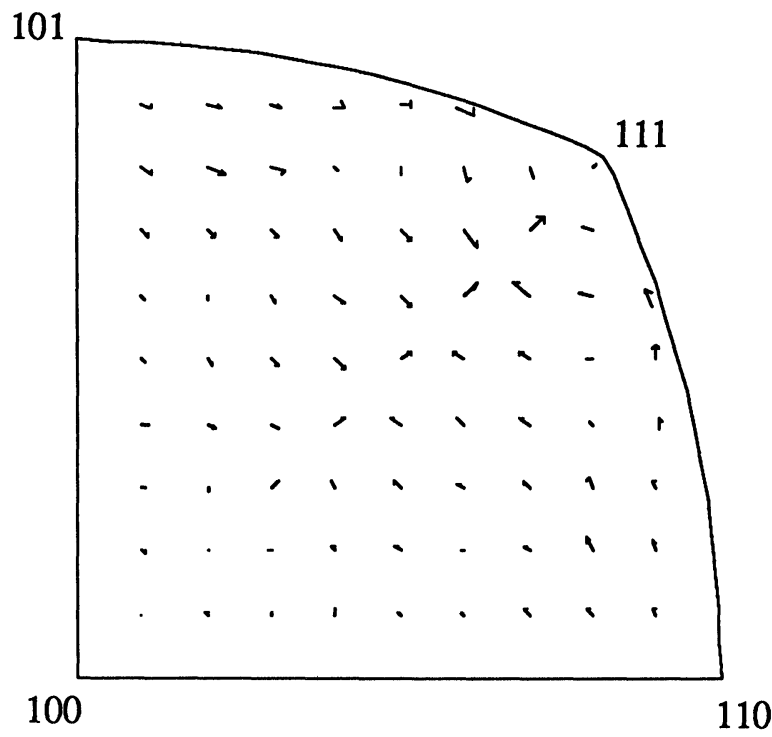

It is remarkable that the flow fields show the same texture development, but the heterogeneous theory shows a slower rate of development, more consistent with experimental observations. 


\section{Summary and Conclusions}

Polycrystalline microstructure is highly stochastic in character, and is best described in the language of probability and statistics. Although some models utilize an explicit description of microstructure, what is of larger significance to engineering properties is the statistical ensemble. The probability density functional of the field $\mathbf{D}(\mathbf{x})$ is a rather comprehensive description of microstructure in the ensemble. Finite-point probability density functions of lattice orientation coherence represent the first few moments of the complete functional description; up to $n=3$ these moments are experimentally realized by ordinary sampling on oblique section planes when a suitable microdiffraction technique is available.

When the bridge between microstructure and tensorial properties is of interest, it is most natural to express microstructural measures in tensorial Fourier form. Basis functions constructed from completely symmetric and traceless tensors exhibit the desired character, and are proposed for representation of microstructures. One advantage of tensorial representations is the geometrical interpretation wherein basis functions are visualized as bundles of lines carrying the lattice symmetry. This construction greatly simplifies the treatment of symmetry in representation theory.

We have illustrated the occurrence of the n-point orientation coherence functions in a theory for creep constitutive behavior in polycrystals. The essential point is that the instantaneous microstructural fields and applied boundary conditions fix the constitutive behavior in the ensemble. Complete statistical information is never available for the ensemble, but the theory enables approximations which utilize finiteorder statistics. The orientation distribution function carries the lowest-order of statistical information about the microstructure, and results in the lowest-order of predictive capability in modeling. Carrying the two-point statistics is shown to give substantial improvements in modeling of creep behavior.

\section{Acknowledgement}

This work was sponsored by the Division of Materials Research of the National Science Foundation. (DMR- 8921304).

\section{References}

1. B. A. Bilby, R. Bullough and E. Smith, Proc. Roy. Soc. Lond., A231, 263 (1955)

2. J. F. Nye, Acta Metall., 1, 153 (1953)

3. E. Kroner, Ergbn. Angew Math., 5, 1 (1958)

4. M. J. Beran, Statistical Continuum Theories (Interscience, New York 1968)

5. B. L. Adams, P. R. Morris, T. T. Wang, K. S. Willden and S. I. Wright, Acta Metall., 35, 2935 (1987)

6. B. L. Adams, T. T. Wang and P. R. Morris, Proc. ICOTOM 8, J. S. Kallend and G. Gottstein eds., (The Metallurgical Society of AIME, 1988), p. 135.

7. P. R. Morris, T. T. Wang and B. L. Adams, Proc. ICOTOM 8, J. S. Kallend and G. Gottstein eds., (The Metallurgical Society of AIME, 1988), p. 157.

8. J. E. Geary and E. T. Onat, Oak Ridge Nat. Lab. Report ORNL-TM-4257 (1974)

9. I. M. Gel'fand, R. A. Minlos and Z. Ya. Shapiro, Representations of the Rotation and Lorentz Groups and their Applications (Pergamon Press, Oxford 1963)

10. R. Courant and D. Hilbert, Methods of Mathematical Physics, vol.1 (John Wiley and Sons, New York, 1953)

11. E. T. Onat and F. A. Leckie, J. Appl. Mech., 55, 1 (1988)

12. H.-J. Bunge, Texture Analysis in Materials Science (Butterworths, London, 1982)

13. J. W. Hutchinson, Proc. Roy. Soc. Lond., A348, 101 (1976)

14. B. L. Adams and D. P. Field, submitted for publication (1990)

15. A. Molinari, G. R. Canova and S. Ahzi, Acta Metall., 35, 2983 (1987) 